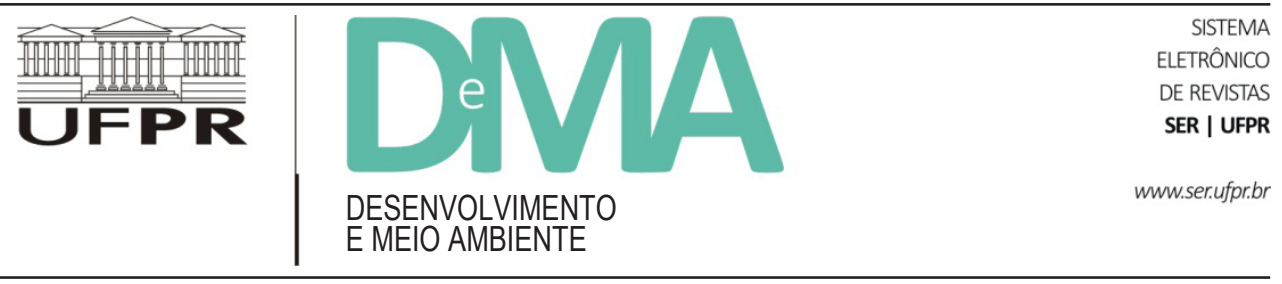

\title{
Experiência de monitoramento participativo a bordo de embarcações da pesca artesanal no Território da Cidadania do Baixo Sul da Bahia, Brasil
}

\section{Participatory Monitoring Experience On-Board Artisinal Fishing Vessels in Território da Cidadania do Baixo Sul da Bahia, Northeastern Brazil}

\author{
Priscilla Nogueira MALAFAIA ${ }^{1 *}$, George $\mathrm{OLAVO}^{2}$, Aline Rocha FRANÇA ${ }^{1}$, Fabiano Silva SEARA ${ }^{3}$, \\ Manoel Bonfim Oliveira FREITAS ${ }^{4}$, João Conceição de ALMEIDA ${ }^{5}$, Sheyla Magalhães de ALENCAR ${ }^{6}$, \\ Lilane Sampaio REGO7 ${ }^{7}$, Marina Siqueira de CASTRO 8,9 \\ ${ }^{1}$ Programa de Pós-Graduação em Modelagem em Ciências da Terra e do Ambiente, Universidade Estadual de Feira de Santana (UEFS), Feira \\ de Santana, BA, Brasil. \\ ${ }^{2}$ Programa de Pós-Graduação em Modelagem em Ciências da Terra e do Ambiente, Universidade Estadual de Feira de Santana (UEFS), Feira \\ de Santana, BA, Brasil \\ ${ }^{3}$ Universidade Estadual de Santa Cruz (UESC), Ilhéus, BA, Brasil. \\ ${ }^{4}$ Associado da Colônia de Pescadores Z-15, Valença, BA, Brasil. \\ ${ }^{5}$ Associado da Colônia de Pescadores Z-17, Camamu, BA, Brasil. \\ ${ }^{6}$ Faculdade São Vicente (ASVIPA), Pão de Açúcar, AL, Brasil. \\ ${ }^{7}$ Núcleo de Etnodesenvolvimento da Empresa Baiana de Desenvolvimento Agrícola (EBDA), Salvador, BA, Brasil. \\ ${ }^{8}$ Empresa Baiana de Desenvolvimento Agrícola (EBDA), Salvador, BA, Brasil. \\ ${ }^{9}$ Universidade Estadual de Feira de Santana (UEFS), Feira de Santana, BA, Brasil. \\ *E-mail de contato: priscillamalafaia@gmail.com
}

Artigo recebido em 17 de março de 2014, versão final aceita em 3 de outubro de 2014.

RESUMO O monitoramento participativo e amostragem biológica realizada por pescadores artesanais, a bordo de suas embarcações, foi conduzida entre janeiro de 2011 a maio de 2012 no Território da Cidadania do Baixo Sul da Bahia. A abordagem adotada teve como objetivo incluir os pescadores no processo de pesquisa, valorizando o conhecimento tradicional e garantindo também a aquisição de dados biológicos de difícil acesso, sobre a desova de peixes recifais capturados em sítios de agregação reprodutiva distantes da costa. A ação foi desenvolvida a partir da integração de pescadores colaboradores no âmbito da pesquisa. Dois mestres da pesca de linha, reconhecidos como especialistas nas comunidades de Valença e Camamu, foram beneficiados com bolsas de pesquisa pela Fundação de Amparo à Pesquisa do Estado da Bahia (FAPESB). Os mestres e tripulação das embarcações consideradas foram qualificados e envolvidos no monitoramento, coletando informações sobre captura, esforço e área de pesca (pesqueiros), realizando biometria, coleta de gônadas e determinação de es- 
tádios de maturação sexual. Os pescadores colaboradores foram acompanhados por pesquisadores e técnicos da Universidade Estadual de Feira de Santana (UEFS) e da Empresa Baiana de Desenvolvimento Agrícola (EBDA). Foram monitoradas 56 pescarias realizadas em 24 pesqueiros, contemplando 3.271 peixes medidos e pesados, pertencentes a 26 grupos taxonômicos populares, além da coleta de 149 gônadas. A avaliação da abordagem foi baseada em parâmetros qualitativos: 1) engajamento e colaboração na atividade e 2) eficácia e coerência na coleta dos dados, preenchimento das fichas e classificação das gônadas. A percepção dos pescadores quanto a relevância do monitoramento participativo para promoção da construção de conhecimentos sobre a atividade pesqueira e da dinâmica reprodutiva dos recursos pesqueiros constitui o principal desafio da abordagem proposta. Uma ação continuada é considerada fundamental para o desenvolvimento crítico individual dos envolvidos e fortalecimento dos laços de confiança estabelecidos entre pescadores, técnicos e pesquisadores, assim como para a indicação de ações que promovam a conservação e uso sustentável dos recursos naturais.

Palavras-chave: pesquisa-ação; observadores de bordo; amostragem biológica; agregações reprodutivas; pesca recifal.

ABSTRACT A pilot experience of participatory monitoring and biological sampling realized by artisanal fishermen on-board their vessels was conducted between January 2011 and April 2012 in a Territory of Citizenship of Southern Bahia. The approach adopted aimed at including fishermen in the research process, valuing traditional knowledge and also ensuring the acquisition of biological data of difficult access on the spawning of reef fish caught in reproductive aggregation sites distant from the coast. The action was developed through the integration of collaborating fishermen within the research. Two masters of the artisanal handline fishing, recognized as experts in the communities of Valença and Camamu, have benefited from research grants by the state foundation for research supporting - FAPESB. The collaborating masters and crews of vessels were qualified and involved in the monitoring process, collecting information on-board about catches, fishing effort, exploited areas (fishing spots), biometrics and stages of sexual maturation. Monthly orientation and monitoring supervision of collaborating fishermen were conducted by researchers and technicians from the State University of Feira de Santana (UEFS) and the agricultural development agency of Bahia state (EBDA). A total of 56 handline fishing operations from 24 fishing spots were monitored; 26 taxa were recorded during these fisheries, 3,271 fishes were measured and weighed, and 149 gonads were collected. The evaluation of the approach was based on qualitative parameters: 1) engagement and collaboration in the activity, 2) efficiency and consistency in data collection, filling out the forms and classification of gonads. The main challenge of the proposed approach is the perception of the fishermen about the relevance of participatory monitoring to promote the construction of knowledge about the fishery and the reproductive dynamics of fisheries resources. A continuous action is considered essential to the strengthening of trust relationships established between fishermen, technicians and researchers, as well as to develop actions that promote the conservation and sustainable use of natural resources.

Keywords: participatory action research; on-board observers; biological sampling; spawning aggregations; reef fisheries.

\section{Introdução}

Os pescadores artesanais constituem importante segmento social entre as populações tradicionais do Nordeste brasileiro, reconhecido pelo estado da Bahia (Bahia, 2013) e amparado por uma Política Nacional de Desenvolvimento Sustentável dos Povos e Comunidades Tradicionais (Decreto Federal No 6.040 de 7 de fevereiro de 2007) (BRASIL, 2007). O monitoramento pesqueiro participativo é considerado uma abordagem estratégica para o desenvolvimento de práticas de gestão comunitária de recursos pesqueiros, através do envolvimento e fortalecimento das comunidades de pescadores artesanais no processo de construção de conhecimentos aplicados à gestão (Berkes et al., 2001; Obura, 2001; Seixas, 2005). 
Entre as vantagens do monitoramento pesqueiro participativo, destaca-se a sua viabilidade para acessar a complexidade das pescarias tropicais e da dinâmica dos recursos pesqueiros em ecossistemas recifais (Bunce et al., 2000; May, 2005) a partir do conhecimento ecológico local atribuído à alta capacidade de observação e experiência acumulada por gerações de pescadores (Junk, 1983 apud Marques, 2012, p. 19; Johannes, 1998). Uma das pescarias artesanais de maior importância socioeconômica e cultural no Brasil é a pesca com linha de mão, realizada em mar aberto e distante da costa, sobre peixes associados aos ambientes recifais da plataforma continental externa e talude superior (Paiva, 1997; Rezende et al., 2003; Costa et al., 2005). Na Bahia, a pesca de linha é atividade econômica secular, estabelecida desde o período colonial (Olavo et al., 2005).

Os principais recursos-alvo da pesca de linha incluem peixes perciformes das famílias dos badejos Mycteroperca bonaci (Serranidae), dos xaréus e olhos-de-boi Seriola spp. (Carangidae) e dos vermelhos (Lutijanidae), como a guaiuba Ocyurus chrysurus, a Cioba Lutjanus analis, o Dentão L. jocu e a Caranha L. cyanopterus (Rezende et al., 2003; Martins et al., 2006). Muitas das espécies das famílias dos vermelhos e badejos apresentam crescimento lento, maturação tardia e comportamento de agregação para a desova do tipo transiente (Domeier \& Colin, 1997), quando todo o esforço reprodutivo anual é concentrado em um período relativamente curto do ano e em sítios específicos, previsíveis no tempo e no espaço. Tais características tornam essas espécies muito vulneráveis à pesca e à degradação de ambientes essenciais para o seu ciclo de vida (Coleman et al., 2000; Olavo et $a l .$, 2011). No entanto, a previsibilidade das agregações reprodutivas favorece o planejamento da conservação desses recursos e o ordenamento pesqueiro focados em épocas e locais específicos, quando a ocorrência do fenômeno é previamente identificada, documentada e validada (Colin et al. 2003).

Dois critérios podem ser utilizados para a identificação de agregações reprodutivas: o registro de um aumento abrupto de densidades ou taxas de capturas (abundância relativa), significativamente superior à média observada em períodos não reprodutivos; e a verificação da desova e documentação in situ (Colin et al., 2003). A validação do caráter reprodutivo das agregações é uma etapa fundamental na pesquisa, sendo realizada através da inspeção das gônadas para verificação de indícios de desova iminente ou desova recente, nos peixes capturados durante pescarias sobre agregações reprodutivas. Porém, a amostragem de gônadas nos desembarques da pesca de linha é bastante dificultada pela necessidade de evisceração dos peixes a bordo, para evitar a deterioração do pescado.

A abordagem adotada no monitoramento participativo aqui reportada teve como objetivo principal incluir pescadores da região do Baixo Sul da Bahia no processo de investigação da pesca sobre as agregações reprodutivas de peixes recifais. Como objetivos específi$\cos$, buscou-se promover a valorização do conhecimento tradicional dos mestres da pesca de linha, além de garantir a aquisição de dados biológicos de difícil acesso sobre a desova de peixes recifais, alvo da pesca de linha em sítios de agregação reprodutiva distantes da costa.

A ação foi potencializada com a implantação de duas bolsas de pesquisa da categoria Pesquisador Local (PL) da Fundação de Amparo à Pesquisa do Estado da Bahia (FAPESB) a mestres da pesca de linha, por meio do Projeto "Desenvolvimento Territorial Sustentável e Multifuncionalidade da Agricultura Familiar nos Territórios de Cidadania da Bahia"1, executado pela Empresa Baiana de Desenvolvimento Agrícola (EBDA), em parceria com o Projeto Pró-Arribada ${ }^{2}$ executado pelo Laboratório de Biologia Pesqueira da Universidade Estadual de Feira de Santana (LABPESCA/UEFS), na área de estudo.

\section{Metodologia}

\section{1. Área de estudo}

A área de estudo está situada na zona costeira e marinha do Território da Cidadania do Baixo Sul da Bahia (Figura 1). Oito municípios costeiros (Jaguaripe,

\footnotetext{
${ }^{1}$ Programa Pacto Federativo / TSC0001/2010 FAPESB/MDA - Núcleo de Etnodesenvolvimento.

${ }^{2}$ Projeto Agregações Reprodutivas de Peixes Recifais no Brasil: subsídio para o licenciamento ambiental de atividades de E\&P (TC n ${ }^{\circ}$ 02001.003030/2001-82IBAMA/ICMBio/FUNBIO).
} 


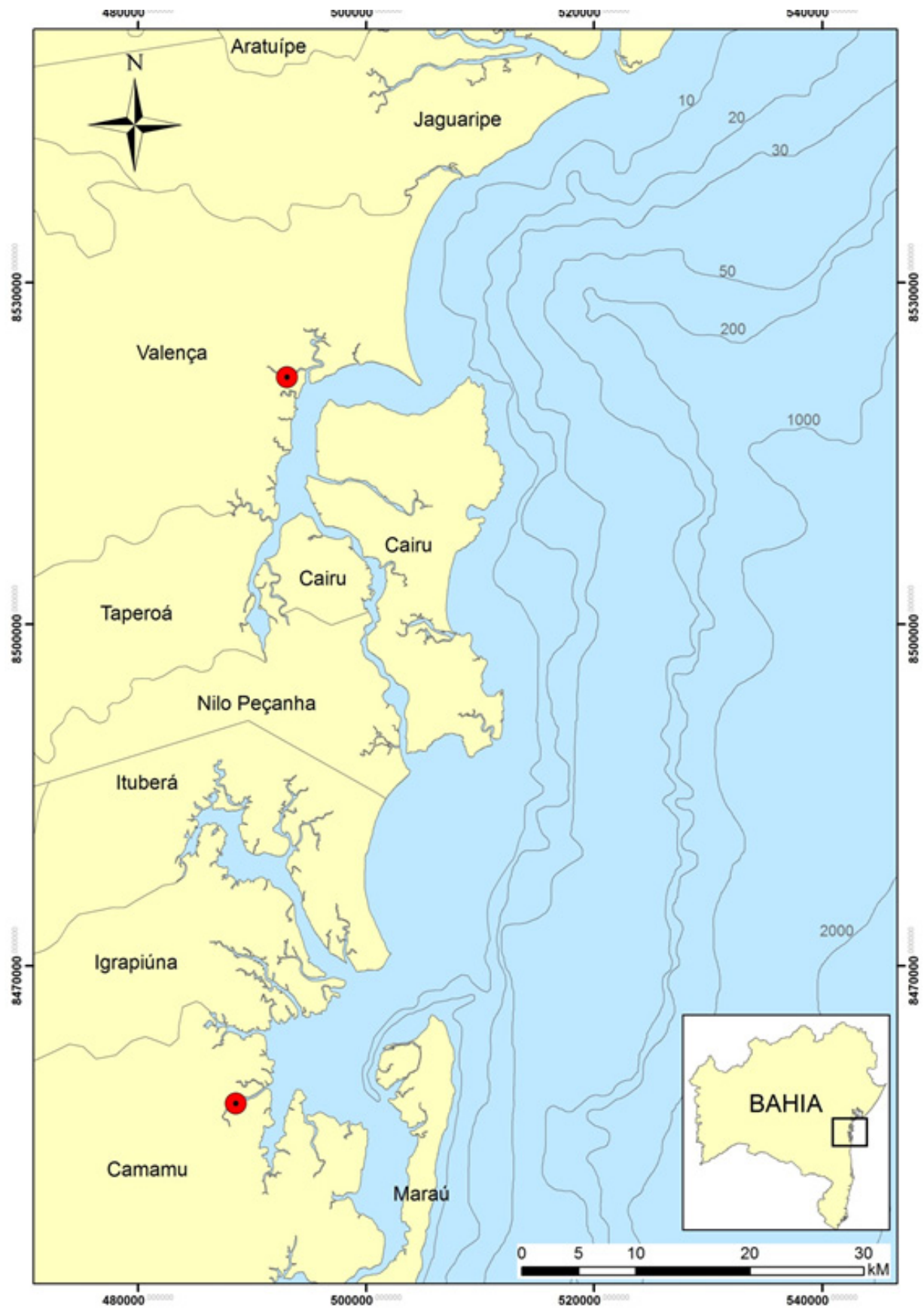

FIGURA 1 - Mapa do Território da Cidadania do Baixo Sul da Bahia, indicando a localização dos municípios costeiros e dos portos de Valença e Camamu, onde estão sediadas as frotas linheiras envolvidas na experiência de monitoramento participativo a bordo das embarcações da pesca artesanal. FONTE: Pró-Arribada (2007). 
Valença, Taperoá, Cairu, Nilo Peçanha, Ituberá, Igrapiúna e Camamu) definem uma região vocacionada para o turismo e atividade pesqueira (Fischer, 2007), tendo como principal centro econômico regional a cidade de Valença. A região é caracterizada por grande biodiversidade e heterogeneidade de ambientes, onde se destaca extenso complexo estuarino, manguezais exuberantes e ecossistemas recifais encontrados desde a linha de costa até a plataforma externa e talude superior (Floeter et al., 2007; Martins et al, 2006). A atividade pesqueira marítima explora essa diversidade regional e está limitada pela pouca disponibilidade de área sobre a plataforma continental, que se apresenta muito estreita, variando entre 5 e 20 milhas náuticas de largura, ao longo de quase toda a costa. A pesca de linha, em particular, está limitada também pela ocorrência e distribuição de ambientes recifais ou fundos de substrato consolidado da plataforma (Costa et al., 2005; Olavo et al., 2005). Os principais recursos-alvo da pesca de linha são, por vezes, capturados em sítios de agregação reprodutiva situados em formações recifais na zona de borda da plataforma, associados a feições do fundo marinho como coroas, paleocanais e ravinamentos na quebra da plataforma (Olavo et al., 2011; Ferreira et al., 2012). As principais frotas pesqueiras artesanais do Baixo Sul da Bahia, que operam em mar aberto, estão sediadas nos portos das comunidades da cidade de Valença, Ilha da Ajuda (município de Jaguaripe), Gamboa, Morro de São Paulo e Boipeba (município de Cairu), Barra dos Carvalhos e São Francisco (Nilo Peçanha) e na sede municipal de Camamu (SEAGRI, 2003).

\subsection{Procedimentos metodológicos}

O monitoramento participativo apresentado neste estudo foi desenvolvido entre janeiro de 2011 e maio de 2012. Sendo realizado o acompanhamento das pescarias e coleta de amostras biológicas a bordo das embarcações, com a colaboração dos próprios pescadores embarcados nas pescarias realizadas por frotas linheiras sediadas no Território da Cidadania do Baixo Sul da Bahia.

A experiência de monitoramento participativo embarcado foi dividida em cinco etapas de execução: (i) identificação das comunidades alvo para o monitoramento e de pescadores colaboradores para atuarem como agentes locais mediante a concessão de bolsas FAPESB/ modalidade Pesquisador Local (PL) ${ }^{3}$; (ii) implantação das bolsas PL e qualificação dos pescadores selecionados; (iii) execução da coleta de dados, amostragem biológica e acompanhamento técnico das atividades de monitoramento participativo; (iv) apresentação dos resultados em reuniões devolutivas nas comunidades envolvidas; e (v) avaliação dos pescadores colaboradores sobre o trabalho desenvolvido.

O trabalho foi iniciado em janeiro de 2011 a partir de visitas preliminares dos técnicos-pesquisadores da EBDA e da UEFS às comunidades de pescadores artesanais da área de estudo, a fim de avaliar as comunidades e potenciais pescadores colaboradores para a implementação do monitoramento pesqueiro embarcado. Para a identificação das comunidades e embarcações das frotas locais para desenvolvimento do monitoramento proposto, foram considerados os portos de origem das principais frotas da pesca de linha, o volume e frequência de desembarques de peixes recifais comerciais (BAHIA, 2003), a receptividade dos pescadores à proposta de pesquisa e a facilidade de acesso aos portos, por terra (rodovias). Os portos selecionados foram os das sedes municipais de Valença e Camamu (Figura 1).

O processo de identificação dos pescadores colaboradores para concessão das bolsas PL ocorreu simultaneamente, durante as visitas realizadas às comunidades no primeiro mês da ação (janeiro de 2011), a partir de contatos com os pescadores de cada uma das comunidades envolvidas. Foram selecionados mestres $^{4}$ de pescaria

\footnotetext{
${ }^{3} \mathrm{O}$ enquadramento das bolsas atribuídas aos pescadores colaboradores segue os requisitos e características das bolsas presentes no Anexo 1 do Edital 007-2010 - Pesquisa ATER - Pacto Federativo, na modalidade denominada Pesquisador Local (PL), destinada a "possibilitar a inclusão de pessoas da localidade impactada pelo projeto proposto. Poderão ser fornecidas bolsas para esses agentes locais caso possam contribuir para as atividades de pesquisa, através do aporte de conhecimentos populares, saberes tradicionais e/ou através de contribuição ao desenvolvimento do conhecimento científico e/ou tecnológico."

${ }^{4}$ Membros da comunidade de pescadores, localmente reconhecidos como detentores de habilidades e conhecimentos específicos das pescarias que realizam e comandam a bordo das embarcações da pesca artesanal. Os "mestres" da tradição da pesca de linha guardam segredos transmitidos
} 
das comunidades de Camamu e Valença, reconhecidos por seus pares como especialistas (Bailey, 1982) na arte da pesca de linha de mão em alto mar.

Os critérios para a escolha dos mestres de pesca foram baseados no tempo de experiência na atividade pesqueira; disponibilidade e interesse em participar do monitoramento; capacidade de registrar informações em fichas de campo; e possibilidade de envolvimento das tripulações de pesca das embarcações sob seu comando. Vale ressaltar que os dois pescadores colaboradores selecionados possuem mais de trinta anos de prática e foram legitimados nas falas de outros pescadores e moradores locais abordados.

As bolsas PL foram implantadas nos meses de fevereiro e março de 2011 a partir do registro dos pescadores colaboradores (bolsistas PL) no Cadastro On-line do Pesquisador no site da FAPESB, elaboração do plano de trabalho juntamente com técnicos e pesquisadores do projeto, e assinatura dos termos de Outorga de cada PL $\left(\mathrm{n}^{\mathrm{o}}\right.$ BOL0224/2011 e $\mathrm{n}^{\mathrm{o}}$ BOL0271/2011).

A qualificação dos pescadores colaboradores (incluindo os mestres e tripulantes das duas embarcações envolvidas) foi diferenciada por embarcação. Em Camamu houve o embarque de um técnico juntamente com o mestre e tripulação de pesca para acompanhamento e observação participante (Berkes et al., 2006) das atividades realizadas durante a pescaria, visando adaptação da rotina de registro de dados, biometria e coleta de amostras biológicas a bordo, assim como o treinamento dos pescadores da tripulação que ficaram responsáveis pelas tarefas do monitoramento. Em Valença, considerando as condições precárias de segurança e trabalho na embarcação, a adaptação da estratégia de monitoramento a bordo e qualificação no processo de registro de dados, biometria e coleta de amostras biológicas a bordo ocorreu no porto, a partir de reuniões entre o mestre da embarcação e o técnico do projeto.

Para cada viagem de pesca monitorada, a coleta de dados a bordo incluiu informação sobre as pescarias, como: áreas de pesca exploradas (pesqueiros visitados), capturas em peso (total e por espécie) e esforço de pesca (dias e número de pescadores), permitindo a obtenção de capturas por unidade de esforço (CPUE, por espécie e por pesqueiro) em unidades de $\mathrm{kg} /$ pescador.dia, considerada como melhor índice de abundância relativa para esta pescaria (Costa et al., 2005). Visando à obtenção de informações acerca da dinâmica reprodutiva e para validação das épocas e locais de ocorrência de agregações reprodutivas das espécies recifais, era também realizada amostragem biológica, incluindo a biometria dos peixes e a coleta de gônadas a bordo. A biometria incluiu a medição do comprimento furcal (CF) e comprimento total $(\mathrm{CT})$ ao centímetro inferior, pesagem individual do peixe e pesagem da gônada, de cada exemplar capturado.

Cada embarcação recebeu um kit de amostragem, incluindo fichas de campo padronizadas, lápis e borracha para registro dos dados; fita métrica e balança dinamômetro manual para a medição e pesagem dos peixes e das gônadas; além de lacres numerados, sacos e potes plásticos para a coleta de gônadas. Os peixes com pesos maiores do que a capacidade da balança manual eram pesados na balança das peixarias, após o desembarque. As gônadas foram pesadas inteiras, acondicionadas em potes plásticos ou bombonas contendo formalina a $10 \%$. As gônadas coletadas foram encaminhadas ao Laboratório de Biologia Pesqueira - LABPESCA/UEFS para tratamento e conservação das amostras.

Durante o desenvolvimento do trabalho, foi incluída a classificação dos estádios de desenvolvimento das gônadas, visto que os pescadores colaboradores já possuíam uma percepção de estádios gonadais, principalmente para fêmeas, coerente com a classificação indicada na literatura científica utilizada (Vazzoler, 1996). Visando reduzir ruídos entre essas duas percepções, foram entregues aos pescadores colaboradores pranchas ilustradas com fotografias para a identificação dos estádios de maturação gonadal.

O acompanhamento técnico e orientação dos pescadores colaboradores foi realizado periodicamente (uma vez ao mês) por técnicos e pesquisadores responsáveis pela avaliação das fichas, recolhimento do material biológico, discussões e esclarecimentos acerca do desenvolvimento do trabalho.

por gerações, principalmente aqueles relacionados à localização de sítios de pesca e comportamento dos recursos pesqueiros, fundamentais para realização do seu trabalho e que lhes confere a "mestrança". 
A apresentação dos resultados ocorreu através de uma reunião devolutiva em cada comunidade pesqueira envolvida. O período da realização desse encontro ocorreu nos dias 04 e 06 de junho de 2012 e contou com a participação de 58 pessoas em Valença e $45 \mathrm{em}$ Camamu, totalizando o montante de 106 participantes. Embora a maioria dos participantes tenham sido pescadores (público alvo), contamos com a presença dos representantes do Serviço Brasileiro de Apoio às Micro e Pequenas Empresas (SEBRAI), Banco do Nordeste do Brasil (BNB), Prefeitura de Camamu e técnicos extencionistas dos escritórios locais da Empresa Baiana de Desenvolvimento Agrícola (EBDA) de Camamu e Valença. A equipe de pesquisa, técnicos e pescadores colaboradores, apresentaram os resultados preliminares do monitoramento pesqueiro embarcado além de registros fotográficos decorrentes da atividade da pesca na região, através da Exposição Fotográfica Nos Ofícios da Tradição Pesqueira, que transpôs em forma de imagens o cotidiano da vida e da faina dos pescadores artesanais das comunidades pesqueiras do Baixo Sul da Bahia.

$\mathrm{O}$ evento apresentou caráter participativo, com a intervenção de membros integrantes das comunidades pesqueiras que colaboraram com a pesquisa, bem como dos pescadores que realizaram o monitoramento das embarcações. Proporcionou o levantamento e discussão de temas sobre a valorização cultural, fortalecimento das comunidades de pescadores e uso sustentável dos recursos pesqueiros e da importância do monitoramento pesqueiro participativo para compreensão do processo reprodutivo e renovação dos estoques pesqueiros, assim como estratégias de manejo e ordenamento das pescarias.

A avaliação da abordagem do monitoramento pesqueiro embarcado se baseou em dois parâmetros qualitativos: i) compreensão do objetivo do monitoramento, engajamento e colaboração na atividade de pesquisa por parte dos pescadores colaboradores; e ii) execução e coerência na coleta dos dados a partir do preenchimento correto das fichas e classificação das gônadas.

\section{Resultados e discussão}

Os resultados serão apresentados e discutidos em dois blocos: (1) avaliação do monitoramento participa- tivo embarcado como ferramenta de geração de diálogo entre o conhecimento científico e os saberes tradicionais e (2) contribuição do monitoramento participativo embarcado para investigação dos indicadores de sítios de agregação reprodutiva.

\subsection{Avaliação do monitoramento participativo embarcado}

\subsubsection{Engajamento e colaboração na atividade.}

A coleta de dados foi realizada de forma continuada, sem interrupções. Os pescadores colaboradores participaram assiduamente dos quatorze encontros realizados com os técnicos-pesquisadores do projeto, assim como do processo de elaboração dos relatórios parciais e finais entregues à FAPESB.

Observa-se que a cooperação estabelecida entre técnicos e pescadores locais abriu caminho para discussões contextualizadas sobre o uso sustentado dos recursos pesqueiros como observado na experiência relatada por Viana et al. (2007), no processo de construção de acordos de pesca para o manejo comunitário do pirarucu na Reserva de Desenvolvimento Sustentável Mamirau.

Os pescadores colaboradores das embarcações envolvidas no monitoramento, tanto em Valença como em Camamu, demonstraram grande interesse e compreensão acerca dos objetivos do estudo, incluindo, além dos mestres, alguns dos seus tripulantes ao longo do desenvolvimento da pesquisa. No caso da embarcação de Camamu, o mestre de pesca dividiu o benefício da bolsa PL com a sua tripulação, assim como as atividades de coleta dos dados e amostragem biológica. Com a embarcação de Valença, embora não tenha havido repartição do valor da bolsa, houve um engajamento relativamente menor dos pescadores da tripulação, embora um agente comunitário formado e vinculado ao Instituto Federal de Educação, Ciência e Tecnologia Baiano (IF Baiano) tenha se engajado na atividade de monitoramento a fim de auxiliar o pescador colaborador. Além das participações na coleta dos dados, os novos colaboradores também se faziam presentes nas reuniões mensais, junto aos pescadores colaboradores e técnico-pesquisadores, enriquecendo as 
discussões com suas experiências sobre a pesca artesanal e os recursos pesqueiros da região.

Observa-se também que o número de tripulantes por embarcação variou de um a quatro pescadores no período da realização do monitoramento embarcado, contribuindo para que essa atividade fosse presenciada por um maior número de pescadores, ampliando o potencial de disseminação das informações e práticas do monitoramento a bordo, entre os pescadores artesanais da região.

Um ponto de destaque, referente ao engajamento na atividade de monitoramento, foi o fato do pescador colaborador (mestre) de Valença dar continuidade à coleta dos dados, mesmo ao término da vigência da bolsa. Segundo ele a experiência do monitoramento pesqueiro foi útil na documentação e compreensão das variações de produção das suas pescarias individuais nas áreas de pesca e épocas do ano, auxiliando no planejamento de suas pescarias futuras.

Segundo Ferreira et al. (2007), Machado \& Mendonça (2007) e Moura et al. (2007) as estratégias de monitoramento participativo e conselhos deliberativos envolvendo pescadores e agentes das comunidades locais constituem importantes ferramentas de subsidio para auxiliar tomadas de decisão no processo de gestão compartilhada e de elaboração de planos de manejo de Unidades de Conservação de Uso Sustentável, como na RESEX do Corumbau, no região do Extremo Sul da Bahia e na APA dos Recifes Costeiros, no litoral de Pernambuco e Alagoas. $\mathrm{O}$ engajamento de comunidades pesqueiras na gestão dos recursos naturais é de fundamental importância (Pinto da Silva, 2007; Kaliskoski et al., 2009; Seixas \& Kaliskoski, 2009 e Seixas et al., 2011), entretanto os desafios para o alcance da gestão compartilhada estão vinculados a diversos fatores, tais como: baixa delegação do poder de decisão à população local (Bernatti et al, 2003; Oliveira, 2003; Kaliskoski \& Saltterfield, 2004; McGrath, 2005); incentivos de participação popular insuficientes (Gutberlet et al, 2004; Seixas \& Troutt, 2004; Thé, 2003; Kaliskoski \& Satterfield, 2004) baixa autoestima e/ou marginalização da população (Gutberlet et al, 2004; Seixas \& Troutt, 2004; Thé, 2003) falta de união e consequentemente de organização local (Camargo \& Petrere Jr., 2004) conflitos entre pescadores locais (Seixas, 2000; Kaliskoski, 2002; Seixas \& Troutt, 2004) falta de suporte técnico e científico no manejo e conservação dos recursos (Benatti et al, 2003; Oliveira, 2003) e o não envolvimento dos usuários dos recursos nas pesquisas científicas (Macedo, 2000; Ruffino, 2001).

\subsubsection{Eficácia e coerência na coleta dos dados, preenchimento das fichas e classificação das gônadas.}

O processo de adaptação dos pescadores colaboradores para a coleta dos dados biológicos e o rigor sobre as informações das pescarias consistiram bons indicadores de analise para o desenvolvimento do monitoramento participativo. As inconsistências encontradas nas 56 fichas preenchidas a bordo puderam ser classificadas em três grupos: erro de coleta, ausência de informação e preenchimento indevido das fichas, como mostra na Tabela 1.

As incoerências no procedimento de coleta estão relacionadas às informações que não faziam sentido nas fichas, registradas de forma incorreta, aparecendo em apenas $18 \%$ das fichas preenchidas. A ausência do registro de informação estava diretamente relacionada à inviabilidade de coleta de dados, observada em $20 \%$ das fichas. O preenchimento inadequado das fichas foi a mais frequente das inconsistências observadas (23\%) e estava relacionada à falta de atenção dos pescadores colaboradores no momento de anotar as informações coletadas.

TABELA 1 - Frequência relativa das inconsistências encontradas nas fichas do monitoramento participativo embarcado preenchidas pelos pescadores colaboradores $(\mathrm{n}=56)$.

\begin{tabular}{lcccccc}
\hline & \multicolumn{2}{c}{ Procedimento de coleta } & \multicolumn{2}{c}{ Registro dos dados } & \multicolumn{2}{c}{ Preenchimentos das fichas } \\
\cline { 2 - 7 } & $\mathbf{n}$ & $\mathbf{\%}$ & $\mathbf{N}$ & $\mathbf{\%}$ & $\mathbf{N}$ & \\
\hline Correto & 46 & 82 & 45 & 80 & 43 & 77 \\
\hline Incorreto & 10 & 18 & 11 & 20 & 13 & 23 \\
\hline
\end{tabular}


As justificativas específicas relatadas pelos pescadores colaboradores sobre as dificuldades encontradas para os registros das informações encontram-se na Tabela 2.

TABELA 2 - Justificativas relatadas pelos pescadores colaboradores sobre as dificuldades encontradas para os registros das informações a bordo nas fichas do monitoramento da pesca.

\begin{tabular}{ll}
\hline $\begin{array}{l}\text { Tipos de informação não } \\
\text { registrada }\end{array}$ & Justificativa \\
\hline Coleta de gônadas & $\begin{array}{l}\text { Peixes abaixo de cinco quilos não } \\
\text { são eviscerados pela tripulação da } \\
\text { embarcação devido às regras lo- } \\
\text { cais de comercialização. }\end{array}$ \\
\hline Comprimentos totais (CT) & $\begin{array}{l}\text { Pescaria com alta captura de indi- } \\
\text { víduos inviabilizava a aquisição } \\
\text { de todos os CT. }\end{array}$ \\
\hline Dados da pescaria & $\begin{array}{l}\text { Esquecimento por parte dos pesca- } \\
\text { dores locais ao preencher a ficha }\end{array}$ \\
\hline
\end{tabular}

Os pescadores colaboradores alegaram que atuar na coleta de dados a bordo e realizar a atividade da pesca simultaneamente, muitas vezes foi algo complicado, pois uma nova rotina foi estabelecida na embarcação, além da faina das pescarias. Dois outros problemas operacionais foram citados sobre a ausência do registro das informações: (1) períodos de chuvas e ventos intensos, os quais dificultavam o manuseio das folhas dos cadernos de campo e exigiam cuidado para com as fichas para não serem danificadas pela chuva e ventos; e (2) baixa iluminação na embarcação durante o período noturno quando era necessário realizar coleta dos dados.

As classificações das gônadas foram realizadas com muita tranquilidade pelos pescadores colaboradores, visto que eles já possuíam uma percepção quanto aos estádios de maturação gonadal, principalmente para fêmeas, bastante similar à classificação científica adotada, seguindo Vazzoler (1996), como mostra a Tabela 3.

O conhecimento "tradicional" geralmente se refere à continuidade cultural transmitida na forma de atitudes sociais, crenças, princípios e convenções do comportamento e de práticas derivadas da experiência histórica (Berkes, 1999). Tais aspectos conferem aos grupos de pescadores artesanais um "repertório de conhecimentos" altamente elaborado quanto aos descritores biológicos do universo que exploram (Marques, 2001; 2012). Os trabalhos de Souza \& Barrella (2001) e Ramires et al. (2007) corroboram a experiência descrita no presente estudo, no que diz respeito ao conhecimento dos pescadores para a determinação do sexo e classificação de estádios de maturação gonadal distintos.

\subsection{Contribuição do monitoramento pesqueiro embarcado para a investigação de indicadores de sítios de agregações reprodutivas de peixes recifais}

Um total de 56 pescarias foram realizadas e 24 pesqueiros foram identificados a bordo das embarcações

TABELA 3 - Tabela de Cognição Comparada. Comparação entre informações dos pescadores colaboradores e citações da literatura científica (Vazzoler, 1996) sobre a classificação e descrição dos estádios de maturação gonadal observados nas fêmeas das espécies alvo do monitoramento participativo.

\begin{tabular}{ll}
\hline Classificação científica & Percepção dos pescadores artesanais \\
\hline I - IMATURO: os ovários são filiformes e translúcidos, de tamanho muito reduzido, loca- “quando a ova tá fininha é porque tá \\
lizados bem junto à parede dorsal, ocupando menos de $1 / 3$ da cavidade celomática; & \begin{tabular}{l} 
verde" \\
\hline II - EM MATURAÇÃO: os ovários ocupam de $1 / 3$ a $2 / 3$ da cavidade celomática, intensa
\end{tabular} Sem correspondência \\
rede capilar, à vista desarmada enxergam-se grânulos opacos (ovócitos) pequenos e mé- \\
dios.
\end{tabular}


de Camamu e Valença envolvidas no monitoramento participativo. A Tabela 4 apresenta as características das pescarias, captura, esforço de pesca, número de pesqueiros e de espécies registrados durante o monitoramento participativo embarcado na região do Baixo Sul da Bahia, entre março de 2011 e maio de 2012. O esforço de pesca variou tanto entre os barcos de pesca quanto nos períodos monitorados. As frequências de pescaria variaram de uma a quatro vezes no mês, com duração de viagem de três a nove dias no mar. O número de pescadores embarcados variou entre um a quatro pescadores por viagem.

Uma síntese dos resultados biológico-pesqueiros obtidos para os principais recursos pesqueiros durante o monitoramento participativo a bordo das embarcações monitoradas em Camamu e Valença é apresentada na Tabela 5. Além da coleta de 149 gônadas um total de
3.271 peixes foram medidos e pesados, classificados a partir do conhecimento tradicional do pescador em 26 grupos taxonômicos populares.

A análise das informações biológicas obtidas nessa amostragem indicou possíveis agregações para seis grupos taxonômicos e suas respectivas áreas de ocorrência. Segundo Colin et al. (2003), taxas de captura três vezes acima da média anual podem constituir indicadores indiretos de agregações reprodutivas de peixes recifais. Picos máximos de CPUE, ultrapassando três vezes a média global do período monitorado foram identificadas para as seguintes espécies: guaiuba $(11,6)$, badejo $(4,8)$, cioba $(6,5)$, dentão $(7)$, caranha $(4,7)$ e xaréu $(4,4)$, respectivamente nas áreas de pesca denominadas de "Calafunda/ Beiradinha", "Canal de Cova da Onça", "Cava da Mata" e "Rego da Caranha" (Tabela 5).

TABELA 4 - Características das pescarias, número de pesqueiros e de espécies registradas durante o monitoramento participativo embarcado na região do Baixo Sul da Bahia, entre março de 2011 a maio de 2012.

\begin{tabular}{|c|c|c|c|c|c|c|c|c|c|c|}
\hline \multirow[b]{2}{*}{ Mês/Ano } & \multicolumn{3}{|c|}{ N. ${ }^{\circ}$ Pescarias Monitoradas } & \multirow{2}{*}{$\begin{array}{l}\text { Média de } \\
\text { Pescado- } \\
\text { res por } \\
\text { Pescaria }\end{array}$} & \multirow{2}{*}{$\begin{array}{l}\text { Média Dias } \\
\text { Pesca por } \\
\text { Pescaria }\end{array}$} & \multirow{2}{*}{$\begin{array}{l}\text { Média Horas } \\
\text { de Pesca por } \\
\text { Pescaria }\end{array}$} & \multirow{2}{*}{$\begin{array}{l}\text { Captura* } \\
\text { Média } \\
\text { por } \\
\text { Pescaria }\end{array}$} & \multirow{2}{*}{$\begin{array}{l}\text { CPUE* } \\
\text { Média } \\
\text { por } \\
\text { Pescaria }\end{array}$} & \multirow{2}{*}{$\begin{array}{l}\mathrm{N}^{0} \text { de } \\
\text { Pesqueiros } \\
\text { Visitados }\end{array}$} & \multirow{2}{*}{$\begin{array}{l}\mathrm{N}^{0} \text { de } \\
\text { Indivíduos } \\
\text { Amostrados }\end{array}$} \\
\hline & $\begin{array}{l}\text { Embar- } \\
\text { cação de } \\
\text { Camamu }\end{array}$ & $\begin{array}{l}\text { Embar- } \\
\text { cação de } \\
\text { Valença }\end{array}$ & Total & & & & & & & \\
\hline $\operatorname{mar} / 11$ & 2 & 1 & 3 & 4 & 9 & 21 & 782,5 & 21,5 & 1 & 113 \\
\hline jun/11 & 2 & 1 & 3 & 3,7 & 6,3 & 17 & 288,33 & 10,5 & 3 & 99 \\
\hline $\mathrm{jul} / 11$ & 1 & 3 & 4 & 3,5 & 4,5 & 8 & 143,55 & 11,1 & 4 & 113 \\
\hline ago/11 & 3 & 1 & 4 & 3,5 & 5,2 & 10 & 153,63 & 7,6 & 3 & 258 \\
\hline set/11 & 2 & 1 & 3 & 3,7 & 6,3 & 5 & 227,62 & 10,2 & 3 & 322 \\
\hline $\mathrm{dez} / 11$ & 3 & 4 & 7 & 3,4 & 3,6 & 9,7 & 239,27 & 17,7 & 6 & 176 \\
\hline $\mathrm{jan} / 12$ & 2 & 2 & 4 & 3 & 6,5 & 9,5 & 640,9 & 33,2 & 2 & - \\
\hline $\mathrm{fev} / 12$ & 2 & 1 & 3 & 3,3 & 5,7 & 10,0 & 295,0 & 15,3 & 1 & 73 \\
\hline $\operatorname{mar} / 12$ & 2 & 2 & 4 & 3,5 & 5,5 & 10,0 & 296,8 & 15,5 & 5 & 83 \\
\hline $\mathrm{abr} / 12$ & 1 & 2 & 3 & 3,3 & 3,7 & 16,7 & 126,6 & 10,5 & 3 & 182 \\
\hline mai/12 & 0 & 3 & 3 & 2,7 & 4,3 & 9,7 & 106,6 & 12,7 & 3 & 533 \\
\hline Total & 29 & 27 & 56 & 3,4 & 5,3 & 12,7 & 317,3 & 15,5 & 24 & 3271 \\
\hline
\end{tabular}

* Captura em kg ; CPUE em kg/pescador.dia. 


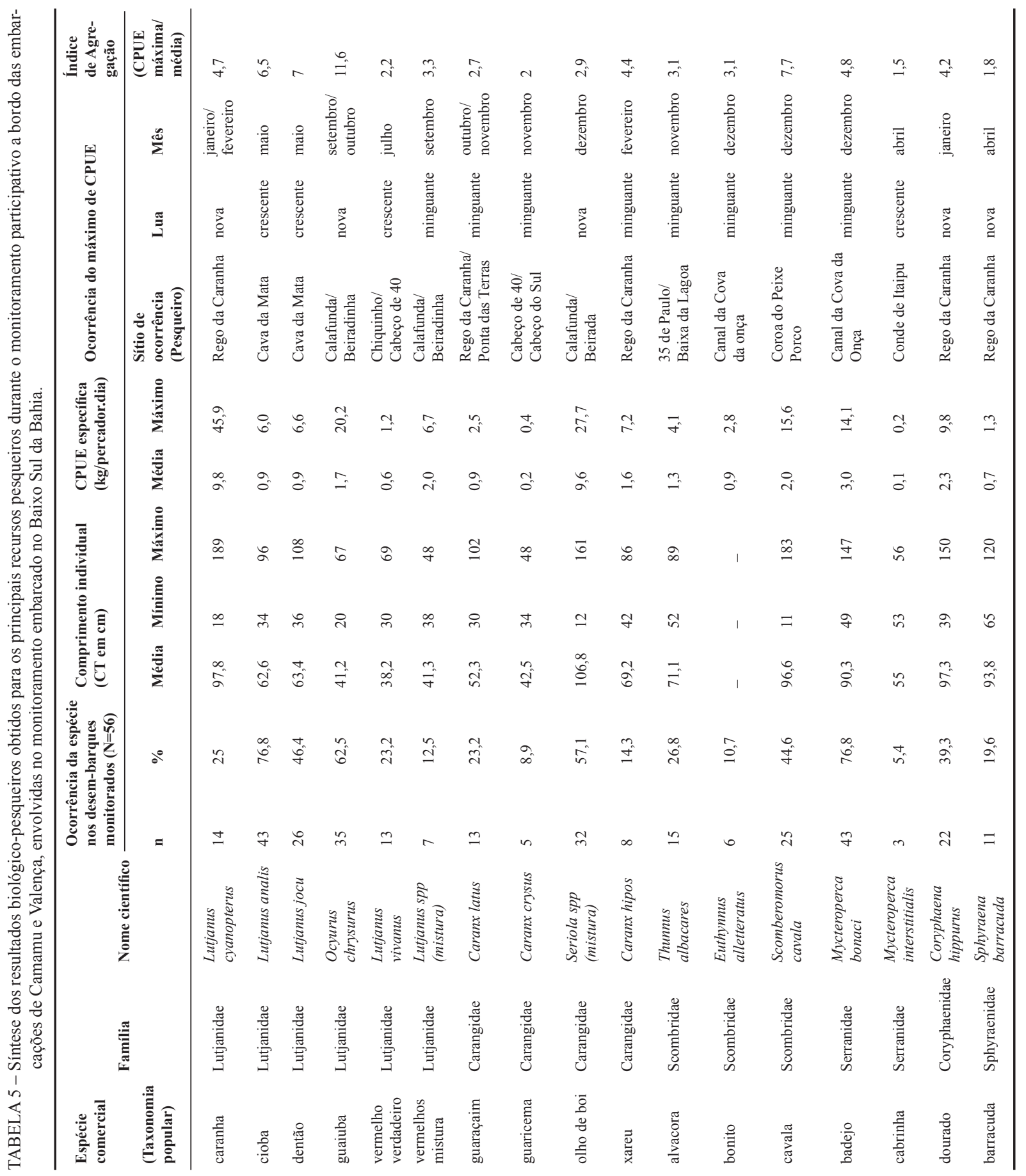


O número de gônadas coletadas para análise em laboratório é apresentado na Tabela 6. Um total de 149 amostras foram obtidas, identificadas por grupo taxonômico popular e por estádio de maturação, sendo todas as gônadas coletadas de indivíduos fêmeas, exceto uma única gônada de caranha macho. O número de gônadas coletadas se mostrou relativamente baixa, quando comparado ao número de indivíduos medidos e pesados, o que pode ser explicado pelo hábito dos pescadores não eviscerarem indivíduos menores do que cinco quilos (5 $\mathrm{kg}$ ) por exigência dos mercados (comerciantes locais, intermediários, donos de peixarias ou comerciantes de outros municípios), impossibilitando a coleta dessas gônadas a bordo.

Os grupos taxonômicos populares de peixes que apresentaram maior número de gônadas coletadas foram: badejo $(n=51)$, cioba $(n=35)$ e caranha $(n=32)$. Dessas gônadas coletadas mais de $93(65,7 \%)$ encontravam-se em estádio gonadal em desenvolvimento (Vazzoler, 1996).

TABELA 6 - Número de gônadas coletadas por grupo taxonômico popular, por estádios de maturação sexual na região do Baixo Sul da Bahia, entre março de 2011 a maio de 2012.

\begin{tabular}{|c|c|c|c|c|c|}
\hline \multirow{2}{*}{$\begin{array}{l}\text { Taxonomia } \\
\text { Popular }\end{array}$} & \multicolumn{5}{|c|}{ Estádio de Maturação (I, II, III, IV) } \\
\hline & $\mathbf{I}$ & II & III & IV & $\begin{array}{l}\text { Não } \\
\text { identificadas }\end{array}$ \\
\hline Badejo & 7 & 12 & 7 & 24 & 1 \\
\hline Caranha & 3 & 11 & 14 & 2 & 2 \\
\hline Cioba & 9 & 15 & 1 & 7 & 3 \\
\hline Dentão & 2 & 3 & 0 & 2 & 2 \\
\hline Guaiuba & 0 & 0 & 0 & 0 & 2 \\
\hline Olho de boi & 1 & 7 & 4 & 0 & 6 \\
\hline $\begin{array}{l}\text { Vermelho } \\
\text { verdadeiro }\end{array}$ & 2 & 0 & 0 & 0 & 0 \\
\hline Total Geral & 24 & 48 & 26 & 35 & 16 \\
\hline
\end{tabular}

O mês de maior frequência de coleta dessas gônadas variou entre os três grupos taxonômicos: a caranha ocorreu principalmente no mês de março $(94,4 \%)$, o badejo em novembro $(92,6 \%)$ e a cioba em maio $(91,7 \%)$ e novembro $(88,9 \%)$, como mostra a Tabela 7 .
TABELA 7 - Meses de maior frequência de coleta das gônadas em estádio de desenvolvimento gonadal avançado para três grupos taxonômicos de peixes recifais.

\begin{tabular}{lllll}
\hline $\begin{array}{l}\text { Taxonomia } \\
\text { Popular }\end{array}$ & $\begin{array}{l}\text { Mês de } \\
\text { maior } \\
\text { coleta das } \\
\text { gônadas }\end{array}$ & $\begin{array}{l}\text { No total de } \\
\text { gônadas } \\
\text { coletadas* }\end{array}$ & $\begin{array}{l}\text { No } \\
\text { gônadas } \\
\text { em ED** } \\
\text { avançado* }\end{array}$ & $\begin{array}{l}\text { Fr gônadas } \\
\text { em ED** } \\
\text { avançado } \\
\text { (\%) }\end{array}$ \\
\hline Cioba & Maio & 12 & 11 & 91,7 \\
Cioba & Novembro & 9 & 8 & 88,9 \\
Caranha & Março & 18 & 17 & 94,4 \\
Badejo & Novembro & 27 & 25 & 92,6 \\
\hline
\end{tabular}

* valores referentes aos respectivos meses apresentados na tabela.

** ED = Estádio de desenvolvimento.

A coleta das informações biológicas pelos pescadores envolvidos nesse estudo gerou indicações inéditas sobre áreas prováveis de ocorrência de agregações reprodutivas de peixes recifais que ainda precisam ser investigadas mais detalhadamente.

Experiências similares quanto a eficiência de práticas de monitoramento participativo da pesca artesanal e coleta de amostras biológicas por pescadores colaboradores e agentes locais qualificados foram também reportadas por Diogo et al. (2006) e Raseira (2007). As parcerias entre pesquisadores e comunidades locais na execução do monitoramento pesqueiro participativo podem reduzir custos de exequibilidade, tornando-se uma ferramenta particularmente útil e condizente com as necessidades dos países em desenvolvimento (Pinto da Silva, 2007).

O refinamento do conhecimento sobre as agregações é animador visto que no Brasil, essas informações ainda são escassas, destacando-se as contribuições pioneiras de Texeira et al. (2004), Peres \& Klippel (2003); do Projeto Meros do Brasil (Gerhardinger et al., 2007) e Projeto Pró Arribada (Freitas et al., 2011; Olavo et al., 2011; Ferreira et al., 2012; Olavo et al., 2013a; Olavo et al., 2013b).

\section{Considerações finais}

O monitoramento participativo embarcado se mostrou uma ferramenta útil tanto para aquisição de dados biológicos quanto para aproximar os pescadores 
da pesquisa cientifica, estimulando um diálogo entre os saberes tradicionais e conhecimento científico.

A experiência do monitoramento participativo embarcado trouxe contribuições iniciais importantes sobre indicação de sítios de agregação reprodutiva potenciais para seis espécies de peixes recifais. Embora ainda em caráter preliminar, esses resultados demandam aprofundamento na investigação dessas ocorrências.

A disponibilização de bolsas de Pesquisador Local potencializou a iniciativa. Porém, a participação qualificada no processo de construção de conhecimentos sobre a atividade pesqueira e qualificação do debate sobre questões relacionadas à gestão participativa e uso sustentável dos recursos naturais depende de ação continuada, fundamental para o desenvolvimento crítico individual

\section{Referências}

Bahia. Secretaria de Promoção da Igualdade Racial - SEPROMI. Direitos dos Povos e Comunidades Tradicionais - cartilha. $2^{\mathrm{a}}$ edição. EGBA: Salvador, Bahia. 84p. 2013.

Bailey, K. D. Methods of Social Research. The Free Press, Macmillan Publishers, Nova York. 1982.

Benatti, J. H.; Mcgrath, D. G.; Oliveira, A. C. M. Políticas públicas e manejo comunitário de recursos naturais da Amazônia. Ambiente \& Sociedade, 5(2), 137-154, 2003.

Berkes, F. R.; Mahon, R; Mcconne, P.; Kalikoski, D.C. (Orgs. da versão em português) Gestão da pesca de pequena escala. Rio Grande: Editora da FURG, 2006.

Berkes, F. Sacred ecology: traditional ecological knowledge and management systems. Taylor \& Francis, Philadelphia and London, UK. 1999.

Berkes, F.; Mahon, R.; McConney, P.; Pollnac, R. B.; Pomeroy, R. S. (Orgs.). Managing small-scale fisheries: Alternative Directions and Methods. International Development Reaserch, Centre, Ottawa, 2001.

Brasil. Decreto $n^{\circ}$ 6.040, de 7 de Fevereiro de 2007. Institui a Política Nacional de Desenvolvimento Sustentável dos Povos e Comunidades Tradicionais. Brasília: DOU de 08/02/2007.

Bunce, L.; Townsley, P.; Pomeroy, R.; Pollnac, R. Socioeconomic manual for coral reef management. Global Coral Reef Management Network, NOAA, IUCN, 2000. dos envolvidos e fortalecimento dos laços de confiança estabelecidos entre pescadores, técnicos e pesquisadores, assim como para a indicação de ações que promovam a conservação dos recursos pesqueiros.

A pesca artesanal caracteriza-se enquanto atividade fundamentalmente importante em seus aspectos sociais, econômicos e culturais para grupos humanos em diferentes momentos históricos e espaços geográficos. Segundo Seixas \& Kalikoski (2009), um requisito para a gestão dos recursos pesqueiros prevê a participação dos pescadores em diálogos entre os setores da pesca, planejamento, implementação e monitoramento das atividades. Todavia, o monitoramento embarcado participativo aqui experienciada configura-se enquanto instrumento preliminar de fomento às bases da gestão participativa.

Camargo, S. A. F.; Petrete Jr. M. Risk analysis applied to the precautionary management of artisanal fisheries in the region of Tucuruí reservoir (Pará, Brazil). Acta Amazonica, 34(3), 473-485, 2004.

Coleman, F. C.; Koening, C. C.; Huntsman, J. A.; Musick, J. A.; Eklund, A. M.; Mcgovern, J. C.; Chapman, R. W.; Sedberry, G. R.; Grimes, C. B. Long-lived reef fishes: The Grouper-Snapper complex. AFS Policy Statement. Fisheries, 25(3), 14-20, 2000. doi:10.1577/1548-8446(2000)025<0014:LRF>2.0.CO;2

Colin, P. L.; Sadovy, Y. J.; Domeier, M. L. (Eds.). Manual for the Study and Conservation of Reef Fish Spawning Aggregations. Society for the Conservation of Reef Fish Aggregations Special Publication, $\mathrm{n}^{\circ} .1$ (Version 1.0), pp. 1-98+iii, 2003. Disponível em: http://webcache.googleusercontent.com/search?q=cache:SaEGU4bjfSIJ:www. researchgate.net/publication/228436345_Manual_for_ the_study_and_conservation_of_reef_fish_spawning_aggregations/file/3deec528510e $82 \mathrm{dc} 7 \mathrm{~b} . \mathrm{pdf}+\& \mathrm{~cd}=1 \& \mathrm{hl}=\mathrm{pt}-$ -BR\&ct=clnk\&gl=br

Costa, P. A. S.; Olavo, G.; Martins, A. S. Áreas de pesca e rendimentos da frota de linheiros na região Central da costa brasileira entre Salvador-BA e o Cabo de São Tomé-RJ. In: Costa. P. A. S.; Martins. A. S.; Olavo. G. (Orgs.). Pesca e potenciais de exploração de recursos vivos na região Central da Zona Econômica Exclusiva brasileira. Rio de Janeiro: Museu Nacional, Série Livros n.13. p. 57-70, 2005. 
Diogo, H. R.; Fraga, A. C.; Figueira, L. D. Programa de gestão compartilhada da pesca artesanal no Baixo-Sul da Bahia. In: Anais do II Seminário de Gestão Socioambiental para o Desenvolvimento Sustentável da Aquicultura e da Pesca no Brasil. Rio Grande do Norte, 29 de nov., 2006.

Domeier, M. L.; Colin, P. L. Tropical reef fish spawning aggregations: defined and reviewed. Bulletin of Marine Science, 60(3), 698-726, 1997.

Ferreira, B. P; Maida, M.; Messias, L. T. Os Conselhos Municipais de Meio Ambiente como Instrumento de Gestão Integrada: A Experiência na Área de Proteção Ambiental Costa de Corais (AL/PE). In: Prates, A. P.; Blanc, D. (Org.). Áreas Aquáticas Protegidas como Instrumento de Gestão Pesqueira. Série Áreas Protegidas do Brasil, 4. Brasília: MMA/SBF. 272p. 2007.

Ferreira, B.; Olavo, G.; Maida, M.; Camargo, J. M.; França, A. R.; Malafaia, P. N.; Maranhão, H.; Baqueiro, C.; Santos, A. Reef fish spawning aggregations on the Northeastern Brazilian coast: status of knowlege and management perspectives. In: Anais do 12th International Coral Reef Symposium. Cairns, Queensland, Australia, 9-13 de jul., 2012.

Fischer, F. (Org.). Baixo Sul da Bahia: uma proposta de desenvolvimento territorial. Salvador: CIAGS/UFBA, 224 p. Coleção Gestão Social - Série Editorial CIAGS, 2007. Disponível em: $<$ http://www.gestaosocial.org.br $>$. Acesso em 11 de fev. 2014.

Floeter, S. R; Ferreira, C. E. L.; Gasparini, J. L. Três Estudos de Caso e Implicações para os Grupos Funcionais de Peixes Recifais no Brasil In: Prates, A. P.; Blanc, D. (Org.). Áreas Aquáticas Protegidas como Instrumento de Gestão Pesqueira. Série Áreas Protegidas do Brasil, 4. Brasília: MMA/SBF. 193-210 p., 2007.

Freitas, M. O.; Moura, R. L.; Francini-Filho, R. B.; Minte-Vera, C. V. Spawning patterns of commercially important reef fish (Lutjanidae and Serranidae) in the tropical western South Atlantic. Scientia Marina, 75(1), 135-146, 2011.

Gerhardinger, L. C.; Medeiros, R. P.; Marenzi, R. C.; Godoy, E. A. S.; Freitas, M. O.; Bertoncini, A. A.; Silva, M. H. Conhecimento Ecológico Local no Planejamento e Gestão de Áreas Marinhas Protegidas e na Conservação de Agregações Reprodutivas de Peixes: A Experiência do Projeto Meros do Brasil. In: Prates, A. P.; Blanc, D. (Org.). Áreas Aquáticas Protegidas como Instrumento de Gestão Pesqueira. Série Áreas Protegidas do Brasil, 4. Brasília: MMA/SBF. 272p. 2007.

Gutberlet, J.; Seixas, C. S.; Thé, A. P. G. Challenges in managing fisheries in the São Francisco watershed of Brazil. In: Biennial Conference International Association for the Study of
Common Property - IASCP, 10, 2004. Proceedings... Oaxaca: Centro Cultural Santo Domingo, 2004.

Johannes, R. The case for data-less marine resource management: examples from tropical nearshore fin-fisheries. Trends in Ecology and Evolution, 13(6), 243-246, 1998. doi:10.1016/ S0169-5347(98)01384-6

Kalikoski, D. C. The Forum of the Patos lagoon: an analysis of co-management arrangement for conservation of coastal resources in Southern Brazil. Vancouver, Tese (Doutorado em Gestão Ambiental e de Recursos Naturais) - UBC, 2002.

Kalikoski, D. C.; Satterfield, T. On crafting a fisheries co-management arrangement in the Estuary of Patos Lagoon (Brazil): opportunities and challenges faced through implementation. Marine Policy, 28, 503-522, 2004.

Kaliskoski, D; Seixas, C. S.; Almudi, T. Gestão compartilhada e comunitária da pesca no Brasil: avanços e desafios. Ambiente \& Sociedade, 12(1), 151-172, 2009.

Macedo, D. S. Manejo Florestal Comunitário: III oficina de manejo florestal comunitário. Manaus: Ibama, 46 p., 2000.

Machado, I. C.; Mendonça, J. T. Gestão Pesqueira Participativa no Complexo Estuarino-Lagunar de Cananéia, Iguape e Ilha Comprida e Área Costeira Adjacente. In: Prates, A. P.; Blanc, D. (Org.). Áreas Aquáticas Protegidas como Instrumento de Gestão Pesqueira. Série Áreas Protegidas do Brasil, 4. Brasília: MMA/SBF. 272p. 2007.

Marques, J. G. W. Etnoictiologia: Pescando Pescadores nas Aguas da Transdisciplinaridade. Revista Ouricuri. 2(2), 9-36, 2012.

Marques, J. G. W. Pescando pescadores: ciência e etnociência em uma perspectiva ecológica. São Paulo: Nupaub, 2001.

Martins, A. S.; Costa, P. A. S.; Olavo, G.; Haimovici, M. Recursos Pesqueiros da Região Central. In: Miguens, A. P.; Tavares, A. R.; Freire, O.; Vieira, R. C.; Jablonski, S. Avaliação do Potencial sustentável de Recursos Vivos na zona Econômica Exclusiva - Programa REVIZEE, Relatório Executivo. MMA; p. 191-216. 2006.

May, D. Folk taxonomy of reef fish and the value of participatory monitoring in Wakatobi National Park, southeast Sulawesi, Indonesia. SPC Traditional Marine Resource Management and Knowledge Information Bulletin, 18, 18-24, 2005. Disponível em: http://www.opwall.com/Library/Opwall\%20library\%20 pdfs/Journal\%20publications/may\%20folk.pdf

McGrath, D. G. Working towards community-based ecosystem management of the lower amazon floodplain. PLEC News and views, 6, 3-23, 2005. 
Moura, R. L.; Dutra, G. F.; Francini-Filho, R. B.; Minte-Vera, C.; Curado, I. B.; Guimarães, F. J.; Oliveira, R. F.; Alves, D. C. Gestão do Uso de Recursos Pesqueiros na Reserva Extrativista Marinha do Corumbau - Bahia. In: Prates, A. P.; Blanc, D. (Org.). Áreas Aquáticas Protegidas como Instrumento de Gestão Pesqueira. Série Áreas Protegidas do Brasil, 4. Brasília: MMA/SBF. 272p. 2007.

Obura, D. O. Participatory monitoring of shallow tropical marine fisheries by artisinal fishers in Diani, Kenya. Bulletin of Marine Science, 69(2), 777-791, 2001.

Olavo, G.; Costa, P. A. S.; Martins, A. S. Caracterização da pesca de linha e dinâmica das frotas linheiras da Bahia. In: Costa, P. A. S.; Martins, A. S.; Olavo, G. (Orgs.). Pesca e potenciais de exploração de recursos vivos na região central da Zona Econômica Exclusiva brasileira. Rio de Janeiro: Editora Museu Nacional, Série Livros, 13. p. 13-34. 2005.

Olavo, G.; Costa, P. A. S.; Martins, A. S.; Ferreira, B. P. Shelf-edge reefs as priority areas for conservation of reef fish diversity in the tropical Atlantic. Aquatic Conservation: Marine and Freshwater Ecosystems, 21, 199-209, 2011. doi: 10.1002/ aqc. 1174

Olavo, G.; Franca, A.; Malafaia, P. N. Indirect Signs of Occurrence of Spawning Aggregations of Dentão Lutjanus jocu (Perciformes: Lutjanidae) in Bahia, Brazil. In: XV Congresso Latino-Americano de Ciências do Mar - COLACMAR 2013. Punta del Este, Uruguai. 27 a 31 de outubro, 2013b.

Olavo, G.; Malafaia, P. N.; Franca, A.; Pereira, M. H.; Seara, F.; Baqueiro, C.; Moreira, J.; Verane, J.; Andrade, L.; Cortesão, J. Sítios de agregação reprodutiva da Caranha Lutjanus cyanopterus (Perciformes: Lutjanidae): Patrimônio da pesca artesanal na Plataforma Continental da Bahia, Nordeste do Brasil. In: XV Congresso Latino-Americano de Ciências do Mar - COLACMAR 2013. Punta del Este, Uruguai. 27 a 31 de outubro de 2013, 2013a.

Oliveira, V. G. Educação ambiental e manejo de recursos naturais em área de proteção ambiental: o caso dos extratores de samambaias da Ilha Comprida - São Paulo. Piracicaba, Dissertação (Mestrado em Recursos Florestais) - Universidade de São Paulo - USP. 2003.

Paiva, M. P. Recursos pesqueiros estuarinos e marinhos do Brasil. Fortaleza: EDUFC, 1997.

Peres, M. B.; Klippel, S. Reproductive biology of southwestern Atlantic wreckfish, Polyprion americanus (Teleostei: Polyprionidae). Environmental Biology of Fishes, 68(2), 163-173, 2003.
Pinto da Silva, P. Da Propriedade Coletiva ao Co-Gerenciamento: Lições da Primeira Reserva Extrativista Marinha Brasileira. In: Prates, A. P.; Blanc, D. (Org.). Áreas Aquáticas Protegidas como Instrumento de Gestão Pesqueira. Série Áreas Protegidas do Brasil, 4. Brasília: MMA/SBF. 272p. 2007.

Pró-Arribada. Projeto Agregações Reprodutivas de Peixes Recifais no Brasil: Subsídio para o Licenciamento Ambiental de Atividades de E\&P. Carteira Fauna Brasil, Programa Termo de Compromisso de Sísmica Marítima, Processo IBAMA n ${ }^{\circ}$ 02001.003030/2001-82, 2007.

Ramires, M.; Molina, S. M. G.; Hanazaki, N. Etnoecologia caiçara: o conhecimento dos pescadores artesanais sobre aspectos ecológicos da pesca. Biotemas, 20(1), 101-113, 2007.

Raseira, M. B. Monitoramento participativo dos acordos de pesca: como avaliara captura de pescado. Manaus, IBAMA/ ProVárzea, 2007.

Rezende, S. M.; Ferreira, B. P.; Fredou, T. A pesca de Lutjanideos no Nordeste do Brasil: histórico das pescarias, características das espécies e relevância para o manejo. Bol, Técn. Cient. CEPENE, 11(1), 257-270, 2003.

Ruffino, M. L. Strategies for Managing Biodiversity in Amazonian Fisheries. In: Blue millennium: managing global fisheries for biodiversity, p. 24. Canadá. 2001.

Seagri. Secretaria de Agricultura. Boletim estatístico da pesca marítima e estuarina - ano 2002. Salvador: SEAGRI / Bahia Pesca. 36 p., 2003.

Seixas, C. S. Abordagens e técnicas de pesquisa participativa em gestão de recursos naturais. In: Vieira, P.F.; Berkes, F.; Seixas, C.S. Gestão integrada e participativa de recursos naturais: conceitos, métodos e experiências. Florianopolis: Secco/APED. Pp 73-105, 2005.

Seixas, C. S. State-property, communal-property or openaccess? The case of Ibiraquera Lagoon, Brazil. In: Anais da Conference of the International Association for the Studies of Common Property - IASCP, Manitoba, 2000.

Seixas, C. S.; Kalikoski, D. C. Gestão participativa da pesca no Brasil: levantamento das iniciativas e documentação dos processos. Desenvolvimento e Meio Ambiente, 20, 119-139, 2009.

Seixas, C. S.; Kalikoski, D. C.; Almudi, T.; Batista, V. S.; Costa, A. L.; Diogo, H. L.; Ferreira, B. P.; Futemma, C. R. T.; Moura, R. L.; Ruffino, M. L.; Salles, R.; Thé, A. P. G. Gestão Compartilhada do Uso de Recursos Pesqueiros no Brasil: Elementos para um Programa Nacional. Ambiente \& Sociedade, 14(1), 23-44, 2011. 
Seixas, C.; Troutt, E. Socio-economic and ecological feedbacks in lagoon fisheries: management principles for a co-evolutionary setting. Interciencia, 29, 362-368, 2004.

Souza, M. R.; Barrella, W. Conhecimento popular sobre peixes numa comunidade caiçara da Estação Ecológica de Juréia Itatins/SP. Boletim do Instituto de Pesca, 27, 123-130, 2001.

Teixeira, S. F.; Ferreira, B. P.; Padovan, I. P. Aspects of fishing and reproduction of the black grouper Mycteroperca bonaci (Poey, 1860) (Serranidae: Epinephelinae) in the Northeastern Brazil. Neotropical Icthyology, 2(1), 1-44, 2004.

Thé, A. P. G. Conhecimento ecológico, regras de uso e manejo local dos recursos naturais na pesca do alto-médio
São Francisco, MG. Tese (Doutorado) - UFSCar. 185 p. São Carlos, SP, 2003.

Vazzoler, A. E. A. M. (Org.). Biologia de reprodução de peixes Teleósteos: Teoria e Prática. Maringá, EDUEM, 1996.

Viana, J. P.; Castelo, L.; Damasceno, J. M. B.; Amaral, E. S. R.; Estupinán, G. M. B.; Arantes, C.; Batista, G. S.; Garcez, D. S.; Barbosa, S. Manejo Comunitário do Pirarucu Arapaima gigas na Reserva de Desenvolvimento Sustentável Mamirauá Amazonas, Brasil. In: Prates, A. P.; Blanc, D. (Org.). Áreas Aquáticas Protegidas como Instrumento de Gestão Pesqueira. Série Áreas Protegidas do Brasil, 4. Brasília: MMA/SBF. 272p. 2007. 